\title{
PAPER
}

\section{The basic hearing abilities of absolute pitch possessors}

\author{
Waka Fujisaki ${ }^{1,2, *}$ and Makio Kashino, ${ }^{2,}$ \\ ${ }^{1}$ Graduate School of Humanities and Sciences, Ochanomizu University, \\ 2-1-1 Ootsuka, Bunkyo-ku, Tokyo, 112-0012 Japan \\ ${ }^{2}$ NTT Communication Science Laboratories, NTT Corporation, \\ 3-1 Morinosato Wakamiya, Atsugi, 243-0198 Japan
}

(Received 2 March 2001, Accepted for publication 25 October 2001)

\begin{abstract}
Generally, people tend to think that absolute pitch possessors or musical experts are superior in all of their auditory and musical abilities. However, not much data has been shown to prove whether any differences exist in the basic hearing abilities of absolute pitch possessors or musical experts for general sounds outside the context of music. In this study, we conducted four experiments to investigate if absolute pitch possessors or music experts are superior in their basic hearing abilities with respect to frequency resolution, temporal resolution, and spatial resolution. In Experiments 1 and 2, we measured frequency discrimination thresholds and thresholds to detect a tone in notched noise to examine the characteristics of frequency resolution. In Experiment 3, we conducted a gap detection task to measure the temporal resolution. Finally, in Experiment 4, we measured interaural time difference discrimination thresholds to examine the spatial resolution abilities. The overall results show that there were no significant differences in frequency, temporal and spatial resolutions among groups with different absolute pitch capability. This indicates that absolute-pitch possessors do not have particularly 'good ears' in terms of resolution.
\end{abstract}

Keywords: Absolute pitch, Basic hearing abilities, Frequency resolution, Temporal resolution, Spatial resolution

PACS number: 43.75.Cd

\section{INTRODUCTION}

Absolute pitch is the ability to identify or produce a pitch of a sound without hearing a reference sound. Generally, people tend to think that absolute pitch possessors are superior in all of their auditory abilities. However, in the field of auditory and music psychology, researchers have focused on the etiology of absolute pitch [1-7] or the merits and demerits of absolute pitch in music cognition [8-12], but have not closely studied the basic hearing abilities of absolute-pitch possessors for general sounds outside the context of music. Studying the basic hearing abilities of absolute pitch possessors may bring insights not only into the mechanisms of absolute pitch itself, but also into problems such as individual differences and the plasticity of auditory functions.

Fundamental elements of auditory abilities include frequency resolution (the ability to detect small frequency differences in sounds), temporal resolution (the ability to

\footnotetext{
*e-mail: hw0805@nifty.com

†e-mail: mkashino@cslab.kecl.ntt.co.jp
}

detect fast temporal changes in sounds) and spatial resolution (the ability to detect small differences in the spatial localization of sounds). The purpose of this study is to investigate whether absolute pitch possessors or music experts are superior in these basic hearing abilities.

Among these fundamental abilities, only frequency resolution has been examined in prior studies of absolute pitch. Oakes (1955) found a positive correlation between pitch-discrimination and pitch-naming abilities, but attributed it to concomitant variations in the amount of musical training that her subjects had received [13]. However, the results did not discount the possibility that the discrimination abilities of absolute pitch possessors were superior. Sergeant (1969) reported conflicting results that no significant relationships between pitch-discrimination and pitch-naming abilities were found [14]. Baggaley (1974) reported general pitch ability and accuracy in piano-note identification were not significantly related, but the accuracy in pure-tone identification and in the general pitch tests were found to be significantly related [15]. However, Baggaley mentioned that no causal relationship between absolute pitch and pitch discrimination should be 
inferred in view of the higher relationships between each of them and the amount of musical training.

Siegel (1972) conducted a pitch discrimination task controlling the musical experience of absolute and nonabsolute pitch possessors [12]. In her first experiment, she found that absolute pitch possessors were significantly better at making sensory discriminations than non-absolute pitch possessors. However, in her first experiment, there were only two subjects in each group. Siegel (1972) conducted a second experiment using a larger sample size (ten absolute pitch possessors and ten controls), and found that possessors of absolute pitch were not more accurate at making sensory discriminations of tone frequency than other musically trained people. However, some subjects in the control group scored more than eighty percent correct on a pitch identification test. Those subjects should be considered as having partial-absolute pitch. When trying to ascertain the effect of the absolute pitch on the pitch discrimination ability, stricter criteria are needed in the screenings of both absolute and non-absolute pitch subjects.

The shapes of the auditory filter also indicate the ability of frequency resolution. The auditory filter is a bank of bandpass filters in the peripheral auditory system, with continuously overlapping center frequencies [16]. To estimate the auditory filter shapes, a paradigm called the notched-noise method has been widely used [17]. A tone is presented in the presence of a masking noise with a spectral notch around the frequency of the tone. Then detection thresholds for the tone are measured with varying the notch width. In this study, we used both the notched-noise method and the frequency discrimination task to investigate whether any differences exist in the frequency resolution among subjects with different musical experience and absolute pitch capability.

Temporal resolution and spatial resolution have not been examined much in the research on absolute pitch. In this study, we chose a gap detection task to examine the temporal resolution among subjects with different musical experience and absolute pitch capability. Broadband noise with a brief temporal gap was presented to the subjects and the minimum detectable gap duration was determined. Lower thresholds indicate better temporal resolution of the auditory system.

To measure spatial resolution among subjects with different musical experience and absolute pitch capability, we conducted an interaural time differences discrimination task. Interaural time differences are a major cue for determing the azimuthal position of a sound. Two tones with different interaural time differences were successively presented, and the minimum difference in interaural time difference necessary to correctly discriminate them was measured. Lower thresholds indicate better spatial resolu- tion.

We conducted four experiments to examine whether any differences exist in frequency, temporal, and spatial resolutions of the auditory system among absolute-pitch musicians, partial-absolute-pitch musicians, non-absolutepitch musicians, and non-absolute-pitch non-musicians.

\section{EXPERIMENT 1}

In this experiment, a frequency discrimination task was conducted to compare the characteristics of frequency resolution among subjects with different absolute pitch capability and musical experience. Frequencies were centered on $1,000 \mathrm{~Hz}$ or $987.76 \mathrm{~Hz}$, which corresponds to $\mathrm{B} 5$ in the equal tempered scale. The reason those two conditions were set was to examine whether some differences exist between the conditions in which frequencies were centered on an in-tune sound and on an out-oftune sound.

\subsection{Method}

\subsubsection{Subjects}

Twenty-seven female musicians and nine female nonmusicians with normal hearing participated in this experiment. Musicians were under-graduate and graduate students of a music college who had had at least eight years of formal musical training before the age of twenty. Nonmusicians were under-graduate and graduate students who had less than two years of musical experience. Their ages ranged from eighteen to thirty-two years old. They were paid for their participation.

Before the main experiments, an absolute pitch test was conducted. Pure tones in three octaves were presented in a random order with the constraint that tones within the same octaves or tones having the same chroma were never presented successively. The duration of each tone was $200 \mathrm{~ms}$. Before conducting the absolute pitch test, it was confirmed that all subjects knew the correspondence between keys of the musical keyboard and musical pitch names. Subjects were asked to identify and choose the musical pitch by clicking buttons (buttons were disposed in the order of musical keyboard) on a computer screen. When subjects could not identify the pitch name of the target sound, they were instructed to choose the key that they thought was closest to the target sound. The next stimulus was presented three seconds after a response. One session contained thirty-six trials, and at least three sessions were conducted for each subject.

Based on the results of the absolute pitch test, subjects were divided into four groups: absolute pitch (AP) musicians, partial AP (PAP) musicians, non-AP (NAP) musicians, and non-AP (NAP) non-musicians. AP subjects were musicians that scored more than $97 \%$ on the absolute pitch test, PAP subjects were musicians that scored 
between $65-85 \%$, NAP(M) subjects were musicians that scored under $25 \%$, and NAP(NM) subjects were nonmusicians that scored under $25 \%$. Eight musicians were diagnosed as AP, seven musicians were diagnosed as PAP, six musicians were diagnosed as NAP(M) and six nonmusicians were diagnosed as NAP(NM). Since the rest of subjects scored between the criteria, their data were excluded from the later analysis.

\subsubsection{Stimuli and equipment}

Two successive intervals of pure tones with different frequencies centered on a test frequency $f_{\mathrm{t}}\left(f_{\mathrm{t}}=1,000 \mathrm{~Hz}\right.$ or $987.76 \mathrm{~Hz}$ ) were used to measure the frequency discrimination thresholds. The signals were $250 \mathrm{~ms}$ in duration, shaped with 10-ms cosine ramps. The level of the stimuli was about $60 \mathrm{~dB}$ SPL. The interstimulus interval was $250 \mathrm{~ms}$. Stimuli were generated digitally and sent to a D/A converter (STAX DAC-TALENT) through a sound card (Digital Audio Labs: Digital I/O Card) on a computer (Gateway 2000 GP6-400). Subjects received binaural signals through headphones (Sennheiser HDA 200) in a soundproof room.

\subsubsection{Procedure}

Frequency discrimination thresholds were measured using an adaptive 2IFC procedure [18], with a two-down one-up stepping rule, which estimates the $70.7 \%$ correct point on the psychometric function. Subjects were presented with two successive intervals of tones and either the first or the second interval was higher than the other. The frequency of the higher tone was $f_{\mathrm{t}}+\Delta f / 2$ ( $\Delta f$ represents the size of the frequency difference) and that of the lower tone was $f_{\mathrm{t}}-\Delta f / 2$. The task of the subject was to indicate the higher interval, and respond by clicking the buttons on the computer screen. If the subject made correct responses on two consecutive trials, the size of the frequency difference was made smaller (divided by 1.4), and if the subject made a wrong response, the size of the frequency difference was made larger (multiplied by 1.4). There were twelve reversal points in one trial, and the points, excluding the first four points, were averaged to determine the threshold. There were three trial sequences in each condition for each subject, and the last two trial sequences were used for the analysis. However, when the thresholds of the last two trial sequences were extraordinarily different (more than three times), two more trial sequences were conducted, and the last two were used for the analysis.

\subsection{Results and Discussion}

Figure 1 shows the mean frequency discrimination thresholds for the four groups, which were analyzed by a two-way analysis of variance. The interaction between groups and conditions $(F(3,23)=3.23, p<0.05)$ was significant. Since the interaction between groups and

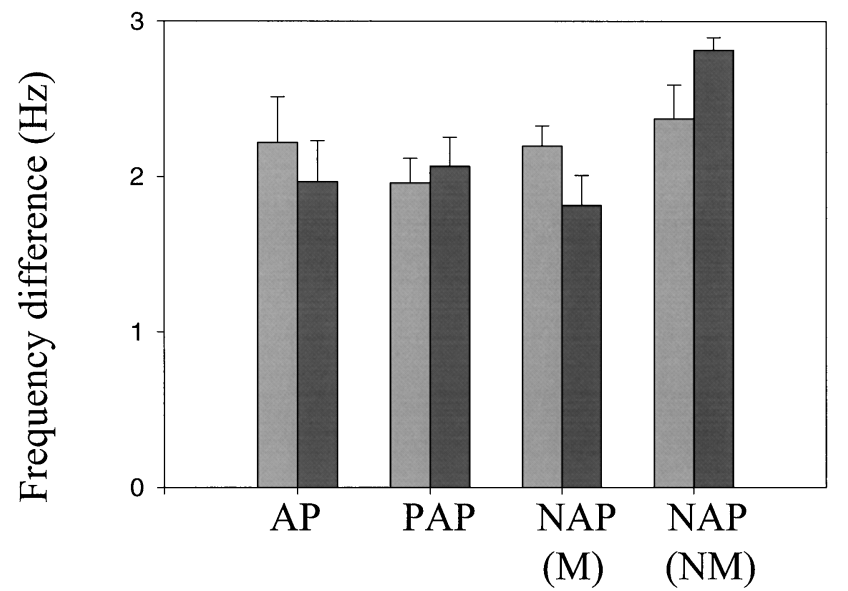

$1000 \mathrm{~Hz}$ $987.76 \mathrm{~Hz}$

Fig. 1 Frequency discrimination thresholds obtained from absolute-pitch musicians (AP), partial-absolutepitch musicians (PAP), non-absolute-pitch musicians (NAP(M)) and non-absolute-pitch non-musicians (NAP(NM)). Black bars are for test frequencies centered on 1,000 Hz. Gray bars are for test frequencies centered on $987.6 \mathrm{~Hz}$ (B5). Error bars represent the standard error.

conditions was significant, simple main effects for each factor were analyzed. When test frequency was $1,000 \mathrm{~Hz}$, there was no significant effect on groups. However, when test frequency was $987.76 \mathrm{~Hz}$, significant effect on groups was found. By Fisher's PLSD test on groups, there were significant differences between AP and NAP(NM) $(p<0.01)$, PAP and NAP(NM) $(p<0.05)$, and NAP(M) and NAP(NM) $(p<0.01)$, indicating no effects of the absolute pitch, but the effects of the musical experiences. This suggests that musical experiences can improve frequency discrimination when test frequency is in-tune to equal tempered scale. Next, the effects of test frequencies on each group were analyzed, but no significant effects were found from all groups.

Absolute pitch capability had no significant effects on the results. This indicates that absolute pitch possessors are not superior in pitch discrimination ability compared to non-absolute pitch possessors, which supports the result of Siegel's second experiment [12]. The interaction of groups and conditions was significant. When test frequency $f_{\mathrm{t}}$ was in-tune to the equal tempered scale, musicians showed better performances than non-musicians. A possible interpretation is that this result reflects the fact that musicians are more trained with in-tune sounds than nonmusicians. Clearly, similar experiments with other test frequencies are needed before generalizing this interpretation.

The frequency discrimination thresholds for a fre- 
quency of $1,000 \mathrm{~Hz}$ obtained in prior studies were about $2 \mathrm{~Hz}$ [19], and our data generally agree with the results of those studies.

\section{EXPERIMENT 2}

The result of Experiment 1 indicates that there are no differences in frequency discrimination thresholds depending on the absolute pitch capability. However, the differences may exist in the shapes of the auditory filter. In this experiment, we measured thresholds to detect a tone in notched noise to examine whether any differences exist in the shapes of the auditory filter depending on musical experience and absolute pitch capability.

\subsection{Method}

\subsubsection{Subjects}

Eight AP musicians, eight PAP musicians, six NAP musicians and six NAP non-musicians participated in this experiment. They were screened by the same absolute pitch test as Experiment 1, and all had also participated in Experiment 1 with the exception of one PAP subject and three NAP non-musician subjects.

\subsubsection{Stimuli and equipment}

Figure 2 shows the stimulus conditions of Experiment 2. A band noise (from 0 to $4,410 \mathrm{~Hz}$ ) having a deep notch was used to mask a tone of $1,000 \mathrm{~Hz}$. The notch was centered on the tone, and the threshold was measured as a function of the distance from the tone to the edge of the noise band $(=\Delta f) . \Delta f$ was varied from 0.0 to 0.4 times the tone frequency $(1,000 \mathrm{~Hz})$. The bandstop filter was an FIR filter with the order of 384 . For example, when the tone frequency was $1 \mathrm{kHz}$ such that $\Delta f$ was $0.3 \mathrm{kHz}$, the masker is composed of two noise bands with flat tops $0.6 \mathrm{kHz}$ wide and skirts that fall $34 \mathrm{~dB}$ in the first $100 \mathrm{~Hz}$ outside the passband.

Two successive intervals of notched noise were

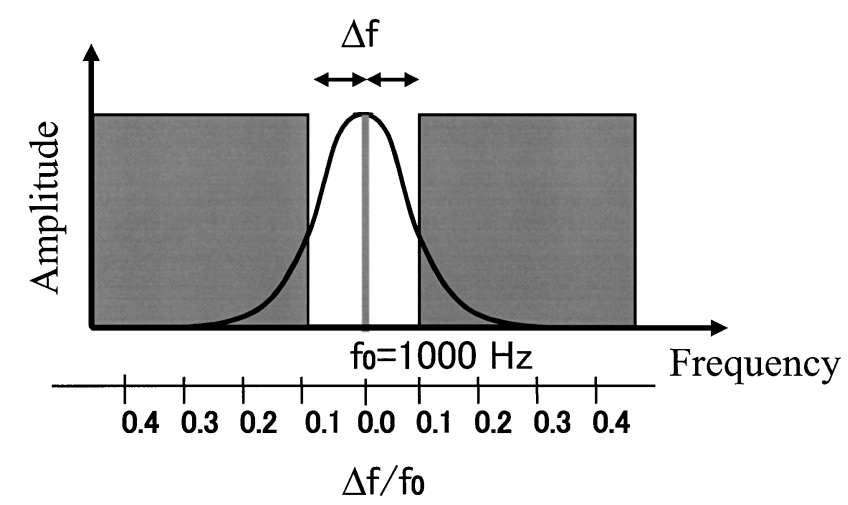

Fig. 2 Stimuli used in Experiment 2. A tone is presented in the presence of a masking noise with a spectral notch around the frequency of the tone. Then detection thresholds for the tone are measured with varying the notch width. presented and either the first or the second interval contained a signal. The duration of the notched noise was $500 \mathrm{~ms}$, and the signal duration was $30 \mathrm{~ms}$ shaped with 5ms cosine ramps. The level of the notched-noise was about $70 \mathrm{~dB}$ SPL. The signal started $460 \mathrm{~ms}$ after the onset of notched noise. The interstimulus interval was $500 \mathrm{~ms}$, and the next trial started 3 seconds after response. Stimuli were generated from the same equipment as in Experiment 1, but presented monauraly.

\subsubsection{Procedure}

Thresholds to detect a tone in the notched noise were measured using an adaptive 2IFC procedure, with a twodown one-up stepping rule, which estimates the $70.7 \%$ correct point on the psychometric function. Subjects were presented with two successive intervals of notched noise and either the first or the second interval contained a signal. The task of subjects was to indicate which interval contained the signal, and to respond by clicking the button on the computer screen.

If the subject made correct responses on two consecutive trials, the level of the signal was decreased by $2 \mathrm{~dB}$, and if the subject made a wrong response, the signal level was increased by $2 \mathrm{~dB}$. There were twelve reversal points in one trial, and the points, excluding the first four points, were averaged to determine the threshold. There were two trial sequences for each subject, and a last trial sequence was used for the analysis.

\subsection{Results and Discussion}

The data for one NAP(NM) subject was excluded from the analysis because of a data recording problem. Figure 3 shows the obtained detection thresholds as a function of $\Delta f$

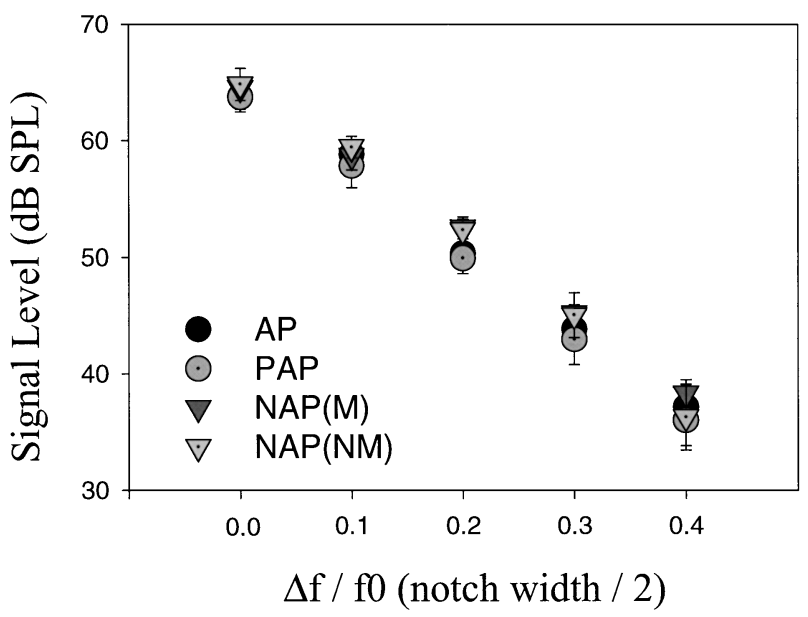

Fig. 3 Threshold signal levels obtained from absolutepitch musicians (AP), partial-absolute-pitch musicians (PAP), non-absolute-pitch musicians (NAP(M)) and non-absolute-pitch non-musicians (NAP(NM)) are plotted as a function of notch width. $\Delta f$ is the distance from the signal to the edges of the noise. Error bars represent the standard error. 
(notch width/2). Mean detection thresholds were analyzed by a two-way analysis of variance for subject groups and the notch width. There were no significant main effect of the subject groups $(F(3,23)=0.55, p=0.66$, n.s. $)$ and interaction between groups and notch width $(F(12,92)=$ $0.26, p=0.99$, n.s.). These results suggest that the shape of auditory filters does not depend on absolute pitch capability nor musical experience.

The thresholds of our subjects were generally higher and slopes were less steep than those of a previous study [17]. A possible interpretation for this is that the shapes of the auditory filter of our subjects were broader than those of Patterson's subjects, but a more plausible interpretation is that our subjects were unfamiliar with this kind of psychophysical experiment.

The results of Experiments 1 and 2, indicate that absolute pitch capability had no significant effects on the results. This suggests that absolute pitch possessors do not have particularly 'good ears' in terms of frequency resolution. In the next experiment, we examined the temporal resolution of the subjects.

\section{EXPERIMENT 3}

In this experiment, we measured gap detection thresholds to examine whether there were any differences in temporal resolution among subjects with different musical experience and absolute pitch capability.

\subsection{Method}

\subsubsection{Subjects}

Eight AP musicians, eight PAP musicians, six NAP musicians and six NAP non-musicians participated in this experiments. All subjects also participated in Experiment 2 .

\subsubsection{Stimuli and equipment}

Two successive intervals of white noise were used as stimuli. The signal level was $60 \mathrm{~dB}$ SPL. The signal duration was $420 \mathrm{~ms}$ with $10-\mathrm{ms}$ cosine ramps. The interstimulus interval was $500 \mathrm{~ms}$. The equipment was the same as in experiments 1 and 2. Stimuli were presented diotically.

\subsubsection{Procedure}

Gap detection thresholds were measured using an adaptive 2IFC procedure, with a two-down one-up stepping rule, which estimates the $70.7 \%$ correct point on the psychometric function.

Subjects were presented with two successive intervals of noise and either the first or the second interval was interrupted to produce the gap. The task of the subject was to indicate which interval contained the gap, and respond by clicking the button on the computer screen. If the subject made correct responses on two consecutive trials, the size of the gap was made smaller (divided by 1.4), and if the subject made a wrong response, the size of the gap was made larger (multiplied by 1.4). There were twelve reversal points in one trial, and the points, excluding the first four points, were averaged to determine the threshold. There were three trials for each subject, and the last two trials were used for the analysis.

\subsection{Results and Discussion}

Figure 4 shows the mean gap-detection thresholds of the four groups. Mean gap-detection thresholds were analyzed by a one-way analysis of variance. No significant differences were found in the gap detection thresholds across the four groups $(F(3,24)=2.60, p=0.07$, n.s. $)$. This suggests that musical experiences or absolute pitch capability do not significantly improve temporal resolution.

The gap detection thresholds obtained in previous studies were about 2-3 ms [20,21], and our data showed almost the same value regardless of the musical experience and absolute pitch capability.

\section{EXPERIMENT 4}

In the last experiment, we measured discrimination thresholds for the interaural time differences (ITDs) to examine whether some differences existed in spatial resolution among subjects with different musical experience and absolute pitch capability.

\subsection{Method}

\subsubsection{Subjects}

Eight AP musicians, seven PAP musicians, six NAP musicians, and six NAP non-musicians participated in this experiment. All subjects also participated in Experiment 1.

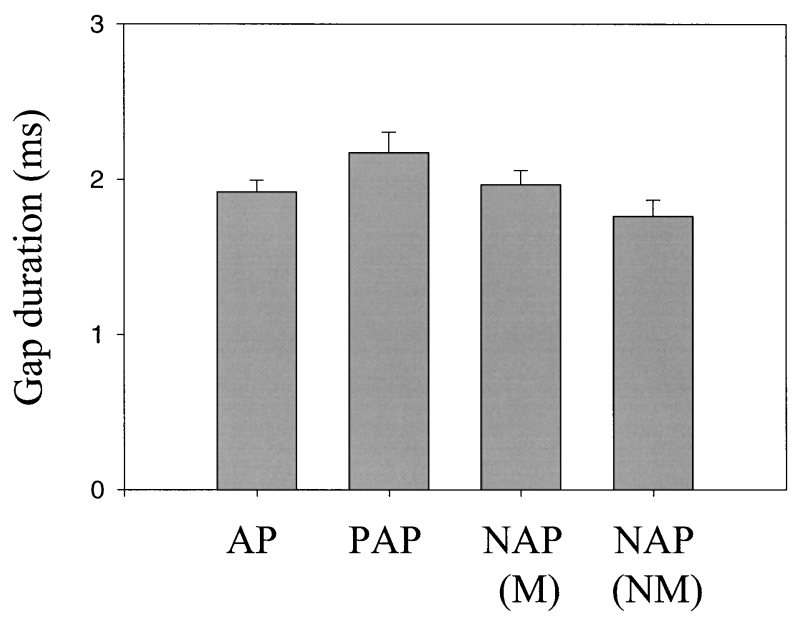

Fig. 4 Gap detection thresholds obtained from absolutepitch musicians (AP), partial-absolute-pitch musicians (PAP), non-absolute-pitch musicians (NAP(M)) and non-absolute-pitch non-musicians (NAP(NM)). Error bars represent the standard error. 


\subsubsection{Stimuli and equipment}

Two successive intervals of pure tones with different interaural time differences were used to measure the ITD discrimination thresholds. The signals were $250 \mathrm{~ms}$ in duration, shaped with 10-ms cosine ramps. The level of the stimuli was about $60 \mathrm{~dB}$ SPL. The interstimulus interval was $250 \mathrm{~ms}$. The equipment was the same as in Experiments 1,2 , and 3 .

\subsubsection{Procedure}

ITD discrimination thresholds were measured using an adaptive 2IFC procedure, with a two-down one-up stepping rule, which estimates the $70.7 \%$ correct point on the psychometric function.

Subjects were presented with two successive intervals of pure tones and either the first or the second interval had larger ITD (A positive value of ITD indicates that the right ear signal leads). The task of the subject was to indicate which intervals were perceived further right, and to respond by clicking the button on the computer screen. If the subject made correct responses on two consecutive trials, the size of the interaural time difference was made smaller (divided by 1.4), and if the subject made a wrong response, the interaural time difference was made larger (multiplied by 1.4). There were twelve reversal points in one trial, and the points, excluding the first four points, were averaged to determine the threshold. There were three trial sequences for each subject, and the last two trial sequences were used for the analysis. However, when the thresholds of the last two trial sequences were extraordinarily different (more than three times), two more trial sequences were conducted, and the last two were used for the analysis.

\subsubsection{Results and discussion}

Figure 5 shows the mean ITD thresholds of the four groups, which were analyzed by a one-way analysis of variance. No significant differences were found in the ITD discrimination thresholds across the four groups $(F(3,23)=1.215, \quad p=0.33$, n.s. $)$. This suggests that auditory spatial resolution, as far as ITD is concerned, does not depend on absolute pitch capability nor musical experience.

There were no significant differences among groups. It should be mentioned, however, that some AP and PAP subjects showed extremely high thresholds of ITD discrimination (up to $187.5 \mu \mathrm{s}$ ) compared to the other subjects (Fig. 6). The ITD discrimination thresholds in ideal conditions are about 5 to $20 \mu$ s [22], but those values may vary with such factors as stimulus duration, stimulus type, ITDs of onset and offset, and the training of the subjects. Therefore, it is not surprising that relatively high discrimination thresholds were obtained in the present experiment, in which short tones with no onset/offset ITDs were used as stimuli and the subjects were not familiar with

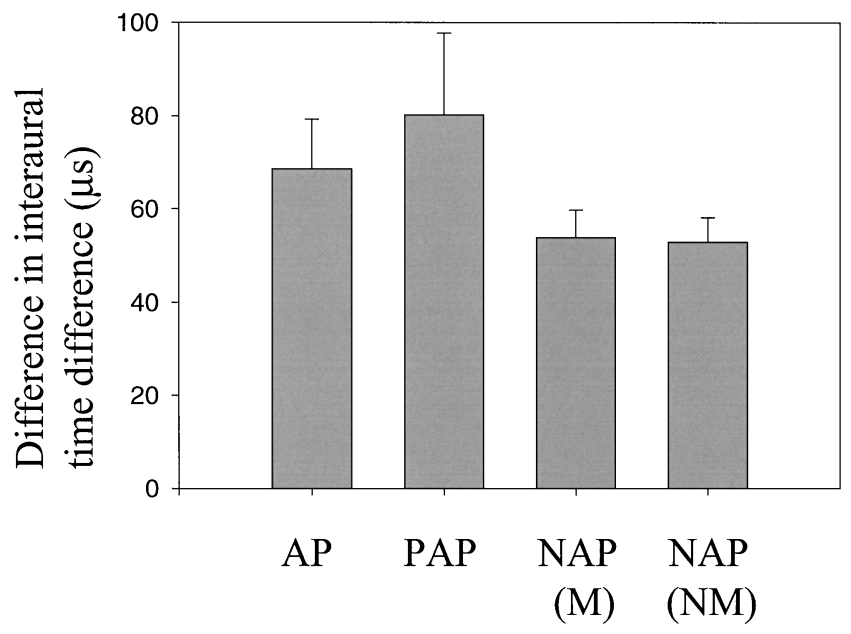

Fig. 5 Interaural-time-difference discrimination thresholds obtained from absolute-pitch musicians (AP), partial-absolute-pitch musicians (PAP), non-absolutepitch musicians (NAP(M)) and non-absolute-pitch non-musicians (NAP(NM)). Error bars represent the standard error.

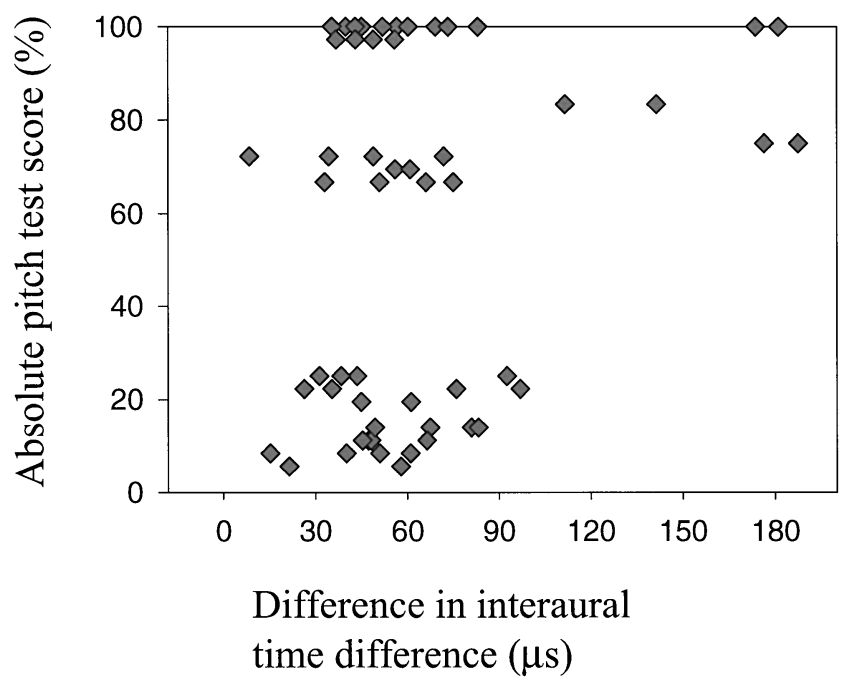

Fig. 6 Scatter diagram of interaural-time-difference discrimination thresholds as a function of the absolute pitch test scores. Two samples from each subject are plotted on this figure.

this type of psychophysical experiment. However, even considering such factors, it is rare that ITD discrimination thresholds exceed $100 \mu$ s for low frequency tones. Since none of the NAP musicians and non-musicians showed such high thresholds, it is possible that some absolute and partial absolute pitch possessors may have difficulty in detecting ITDs or in spatial processing in general. Further studies with a larger number of subjects are needed in order to clarify this point.

\section{GENERAL DISCUSSION}

In this study, we conducted a series of experiments to 
examine whether musical experience and absolute pitch capability has any influence on basic hearing abilities. In Experiment 1, we measured frequency discrimination thresholds to see the characteristics of frequency analysis. In Experiment 2, we conducted the signal detection task using the notched-noise paradigm to evaluate the shapes of auditory filter that also represents the ability of frequency resolution. In Experiment 3, we conducted the gap detection task to measure the temporal resolution of the auditory system. Finally, in Experiment 4, we measured interaural time differences discrimination thresholds to examine the abilities of spatial resolution. The overall results show that there were no significant differences in frequency, temporal and spatial resolutions among groups with different absolute pitch capability.

Absolute pitch possessors tend to be thought of having a 'good ear', but our findings show that absolute pitch possessors do not have particularly 'good ears' in terms of frequency, temporal and spatial resolutions at least for the tasks in the present study.

The next question is, then, where do the differences exist between absolute and non-absolute pitch possessors? Since absolute pitch is the ability to identify musical pitch, it is possible for differences from non-absolute pitch to exist in mechanisms directly related to musical pitch perception. Further studies are needed that examine what information is used by absolute pitch possessors when they identify musical pitch and how their usage of this information differs from how non-absolute pitch possessors use it.

\section{REFERENCES}

[1] P. T. Brady, "Fixed-scale mechanism of absolute pitch", $J$. Acoust. Soc. Am., 48, 883 (1970).

[2] L. L. Cuddy, "Practice effects in the absolute pitch judgment of pitch", J. Acoust. Soc. Am., 43, 1069 (1968).

[3] L. L. Cuddy, "Training the absolute identification of pitch", Percept. Psychophys., 8, 265 (1970).

[4] M. Meyer, "In the memory of absolute pitch capable of development by training?", Psychol. Rev., 6, 514 (1899).

[5] J. Profita and T. G. Bidder, "Perfect pitch", Am. J. Med. Genet., 29, 763 (1988).

[6] A. Van Krevelen, "The ability to make absolute judgments of pitch", J. Exp. Psychol., 42, 207 (1951).

[7] C. H. Wedell, "The nature of the absolute judgment of pitch", J. Exp. Psychol., 17, 485 (1934).

[8] A. Bachem, "Time factors in relative and absolute pitch determination", J. Acoust. Soc. Am., 26, 751 (1954).

[9] A. Benguerel and C. Westdal, "Absolute pitch and the perception of sequential musical intervals", Music Percept., 9, 105 (1991).

[10] K. Miyazaki, "Absolute pitch as an inability: Identification of musical intervals in a tonal context", Music Percept., 11, 55 (1993).

[11] K. Miyazaki, "Perception of relative pitch with different references: Some absolute-pitch listeners can't tell musical interval names", Percept. Psychophys., 57, 962 (1995).

[12] J. A. Siegel, "The nature of absolute pitch", in Studies in the Psychology of Music, I. E. Gordon, Ed. (University of Iowa Press, Iowa City, 1972), Vol. 8, p. 65.

[13] W. F. Oakes, "An experimental study of pitch naming and pitch discrimination reactions", J. Genet. Psychol., 86, 237 (1955).

[14] D. C. Sergeant, "Experimental investigation of absolute pitch", J. Res. Music Educ., 17, 135 (1969).

[15] J. Baggaley, "Measurement of absolute pitch: A confused field", Psychol. Music, 2, 11 (1974).

[16] H. Fletcher, "Auditory patterns", Rev. Mod. Phys., 12, 47 (1940).

[17] R. D. Patterson, "Auditory filter shapes derived with noise stimuli”, J. Acoust. Soc. Am., 59, 640 (1976).

[18] H. Levitt, "Transformed up-down method in psychoacoustics", J. Acoust. Soc. Am., 49, 467 (1971).

[19] C. C. Wier, W. Jesteadt and D. M. Green, "Frequency discrimination as a function of frequency and sensation level", J. Acoust. Soc. Am., 61, 178 (1977).

[20] R. Plomp, "Rate of decay of auditory sensation", J. Acoust. Soc. Am., 36, 277 (1964).

[21] M. J. Penner, "Detection of temporal gaps in noise as a measure of the decay of auditory sensation", J. Acoust. Soc. Am., 67, 608 (1977).

[22] R. G. Klumpp and H. R. Eady, "Some measurements of interaural time difference thresholds", J. Acoust. Soc. Am., 79, 859 (1956).

Waka Fujisaki was born in 1973. She received B.A. from Waseda University in 1997, and M.A. from Ochanomizu University in 1999. She is presently a doctoral student at Ochanomizu University and a special trainee at NTT Communication Science Laboratories. She is a member of the Acoustical Society of Japan, the Japanese Psychological Association, and the Japanese Society for Music Perception and Cognition.

Makio Kashino was born in 1964. He received B.A., M.A., and $\mathrm{Ph} . \mathrm{D}$. in experimental psychology from the University of Tokyo. He joined NTT Basic Research Laboratories in 1989, and currently a senior manager of research planning section of NTT Communication Science Laboratories. He was a visiting scientist at the University of Wisconsin in 1992-93. His expertise is in psychophysics and cognitive neuroscience, especially of auditory perception. 